\title{
A Brief Study on Aquaponics: An Innovative Farming Technology
}

\author{
Amith A. Kulkarni, Dhanush P., Chethan B. S., Thamme Gowda C.S. \\ and Prashant Kumar Shrivastava* \\ Department of Mechanical Engineering, Vidyavardhaka College of Engineering, Mysuru, Karnataka, India; \\ Amith.kulkarni.54@gmail.com, Dhanushp147@gmail.com, Chethanbs050@gmail.com, \\ Thammegowda.CS@vvce.co.in, er.prashant1986@gmail.com
}

\begin{abstract}
Objective: The article presents a discussion on the aquaponics technology, including its working principles and applications in farming as well as the trends and challenges associated with its use in different areas of agriculture. Methods/statistical analysis: In this work, we discuss aquaponics, a combination of two techniques: a. 'hydroponic', which means cultivating plants/vegetables without soil, and b. 'aquaculture', which means fish farming. Aquaponics has become a popular technique because of its similarities to natural ecosystem, where the only difference is that it is a controlled system, in which the fish eats and produce ammonia, beneficial bacteria convert ammonia produced by the fish into nutrients, and the plant absorbs the natural fertilizer and the nutrients. In addition, the water carrying the ammonia and nutrients is continuously recirculated through the system. Findings: The present study was conducted to explore and find a better way to apply the aquaponics agriculture system in various agriculture industries. Findings show that this method enhances the benefits and eliminates the many drawbacks that occur in traditional soil-based agriculture. Application/improvement: This agriculture system does not depend on the soil, and no pesticide is required during the farming. This system is suitable for year-round farming and can produce high-quality vegetables at a much higher yield rate. Vegetables grow at a much faster rate using this technique, compared traditional farming techniques. Aquaponics takes place in a closed system and there is no discharge of waste into the stream. This system produces up to $30 \%$ more production compared to traditional farming using the same amount of space. This system requires less water compared to traditional farming.
\end{abstract}

Keywords: Automation, Aquaponics, Aquatic Farming, IoT, Arduino, Aquaculture

\section{Introduction}

The word aquaponics is the combination of 'aqua' and 'ponics'; aqua denotes aquaculture, where fish are raised in a controlled environment, and 'ponics' is a Latin word, which means 'to work', and cultivation is carried out in soilless media. Nowadays, population's food demand has grown manifold and indeed become a crisis, with regular agricultural farming barely meeting all the requirements, and severe problems faced by farmers, such as high cost of fertilizers, water for irrigation, land for farming, and so on. To overcome these problems a new technique called aquaponics comprising of automation and which mimics natural ecosystem has been introduced. The method is very efficient, cost-effective, inexpensive, and free of various other issues associated with traditional agriculture; this being a modern, computer-driven technique, there is enormous scope for automation and, thus, a wide range of applications in agriculture. Aquaponics combines regular agricultural methods with aquaculture. In this system, the fish cultivated eats and produces waste, which in turn is used as the perfect fertilizer for growing the required crop. Aquaponics describes the relationship between the water, aquaculture design, and nutritional values. Plants grow in watercourses and circulates the power through the bio-integration various components. Hence, these are very beneficial for the food system, and in most of the

${ }^{*}$ Author for correspondence 
food crops obtained from the regular farming heavy-duty pesticides are used that cause serious health hazards. Thus, aquaponics is highly prescribed, and the control system of the aquaponics can be heavily automated to yield more quantity of agricultural produce or plants and vegetables. 1 The schematic diagram of the aquaponics system is shown in Figure 1.

\section{Literature Survey}

The quality of soil and climatic condition differ in various regions. Some soils have different structures with substantial content clay or sand. Some soils have concrete, asphalt, and also heavy amount of chemicals and fertilizers used during cultivation, and this coupled with water erosion, wind erosion, and other natural calamities leads to serious health hazards to food consumers. $\underline{2}$

However, these issues are resolved in aquaponics as it eliminates weeds and the system recycles the water which is rich in nutrients. There is no toxic run-off in the system. Aquaponics uses just 10\% of total water used in regular agricultural farming. Hence, it is very effective and useful for drought-prone areas. 3 Aquaponics uses a hydroponics system that consists of a. a fish tank - to house the fish and grow beds in which the plants are grown; b. a pump, to move water from the lowest part of the bed to the highest part of the bed; $c$. a pipe, through which water is recirculated; $d$. an air pump ensures adequate oxygenation of water; e. a filter tank installed removes solid wastes before water reaches the hydroponic grow bed; f. a sump tank is kept at the lowest part where water collects and the water-circulation pump is located; and g. bioreactor, which provides mechanical and biological filtration and plant root support in drained grow beds. $\underline{4}$

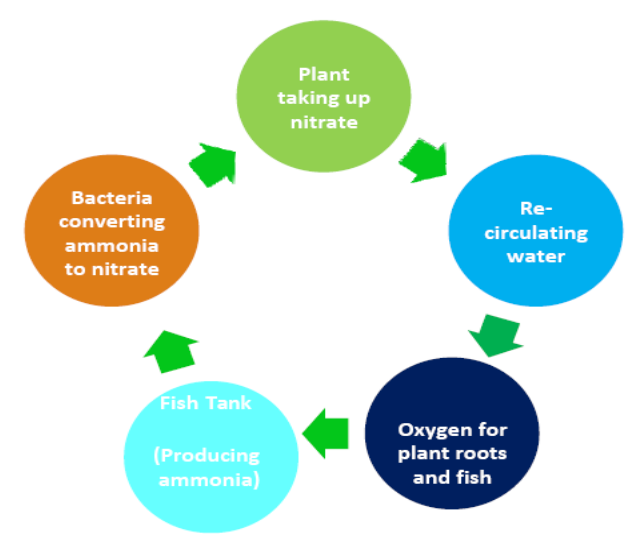

Figure 1. Schematic diagram of the aquaponics system.

\subsection{Types of Aquaponics Systems}

\subsubsection{Floating Raft or Deep Water Culture}

Plants are grown with the pieces of Styrofoam, which glide on the exterior of water-filled grow beds.

\subsubsection{Flood and Drain}

Plants are grown in an intermediate-filled grow bed. The substrate medium acts as a biological as well as mechanical filter, which helps with better maintenance of plants cultivated.

\subsubsection{Nutrient Film (NFT)}

Plants are grown in pipes through which a small amount of water is continually flowing; water is propelled constantly from the tank to the filter. $\underline{5}$

The use of geothermal energy for food production in urban areas has also gained popularity, as this concept uses excavated water to heat and cool buildings. The greenhouse gases along with the combination aquaculture system exhibit a demand curve that is totally different from the regular heating technologies. Thus, compared to conventional greenhouse gases and by using deep water culture grow beds, we can use thermal buffers to achieve peak loads within the prescribed limit of zone temperatures. However, given the drawback that the gradient fish tank temperature maximizes, which may kill the plants, further research is required to develop the technology and devise/implement appropriate design changes to take care of nibbling issues and make the system more robust and capable of protecting the aquaculture as well as the plants being cultivated. $\underline{6}$

In the next step, a laboratory analysis of the aquaponics system was conducted using the PLC AND Lab VIEW, and data were collected about the various parameters in the system, including water level and water temperature. Water temperature is measured through various automated sensors and transducers, and the overall control is done through the PLC. Experimental simulations were carried out in Lab VIEW, Siemens S7-1500 PLC is used for data acquisition, and Lab VIEW is also used to develop a userfriendly interface to display the required data. $\underline{7}$

Now, the new big thing in ICT (Information and Communication Technology) called Internet of Things (IoT) allows to store the data in the Cloud (Cloud Computing/Cloud Storage) that can be accessed virtually from anywhere, as all data are stored in remote servers 
and not at physical servers at the user's location; usually, Cloud data are used to store only historical data and not real-time data; hence, data acquired from the Cloud is not taken into consideration for instant actions. To address this issue, a fog server is introduced between the device and the Cloud, and the server can react based on the real-time data gathered from the device. Therefore, for any analysis, the user can directly approach the fog server for real-time data. In regards to the context of our study, the aquaponics grow bed requires real-time dataacquiring devices to carry out more decisive acts; for example, the water level analysis can be done easily by the fog server, as the integration of multi-trophic aquaculture is crucial for exerting effective control over water flow. Successful control of siphoned water movement depends on receiving accurate real-time inlet and outlet flow rate of the water relayed by the sensors installed in the system and, most importantly, on instantaneous data acquisition from the fog server. $\underline{8}$

Moreover, aquaponics NFT system requires continuous flow of water. Hence, the monitoring and controlling of water flow is a very important aspect. The NFT is situated between the hydroponic and the aquaculture. Since water carries ammonia and various nutrients, an advanced system is required to more precisely and efficiently control and monitor all these parameters, including temperature level and $\mathrm{pH}$ level. This can be done by using the IoT integrated with smartphones. IoT-driven apps can also be used to investigate plant growth, such as plants' height, weight, and so on. Various types of sensors are used for measuring the said parameters, including temperature, water level, $\mathrm{pH}$, humidity, and ammonia content. By implementing this system, a more advanced and efficient monitoring and controlling of the said parameters is possible, which in turn will immensely benefit and boost the growth of plants. 9

Thereafter, in indoor aquaponics system, parameters such as temperature, humidity, and soil moisture are controlled through hardware platforms combined with the IoT. Both modes of IoT, that is, automatic and manual, are used in the process. In the automatic mode, the system can be controlled through the mobile application and requires no user intervention. The system is completely monitored by sensors and a control system with the manual average speed of 5 seconds based on the network traffic and network conditions; this type of communication system is dependent on Internet speed. In the manual mode, a user regularly intervenes to monitor parameters, and the communication system in this case is totally controlled by the IoT. $\underline{10}$

In automated indoor aquaponics cultivation technology, an auto feeder is placed which feeds the fish with the marketable pelleted feeds comprising 30\% of crude containing protein. This will supply the plants the required nutrients that aid their growth. An automatic filter works to filter and eliminate waste from the water before it gets to the grow bed again through the process of recirculation. A method called Arduino function is used, which acts as the brain of the system, and the inputs from Arduino is collected by the sensors and output is converted into action carried out by an the actuator. This back-and-forth input and output is a form of feedback mechanism and a closed-loop system, which allows for optimal control and integration of the controller with the rest of the system components. Actuators work efficiently in a closed-loop control system. $.11, \underline{12}$

Another method to monitor and control environmental parameters is the use of raspberry pi as the network backbone, which provides proper nurturing conditions for the fish and the plants and keeps the parameters in the right balance to help with the correct way of growth of the plants and fish. A central host is provided by the controller which gets the input from the sensors and transducers. The readings are compared to set the desired points and later corrected with the error through comparisons and backed up by the raspberry pi networks. Then, based on the comparisons and controller decisions, the output produced by the actuators can be triggered by the automatic mode and the manual mode. Subsequently, the backup network hosts the readings of the sensor on the display for the analysis of the data logs. The network also allows the user to see the data logs anywhere and manually control the log parameters such as the rate of dissolved oxygen, temperature, and $\mathrm{pH}$ values. $\underline{13}$

\section{Results and Discussion}

Agriculture is vital process and source for food generation for the humankind. The rate of food consumption increasing day by day, but the supply and growth aren't simply enough to meet the growing food requirements, and this short supply occurs on account of several factors. Thus, to overcome the problems of the low yield in agriculture, an innovative, cost-effective, and efficient way of farming that operates based on a simple mechanism 
is used. The present study's findings concerning past research literature and findings show that water in fish tanks gets contaminated with fish excretion; this produces a large amount of ammonia, necessitating flushing out water at a rate of $10-20 \%$ of the entire amount of the tan every day. In general, impure water is often pumped out to flow into open streams; as a result the harmful atmosphere created by the presence of impure water, fish are exposed to diseases. Therefore, it is necessary that fish be treated frequently with medicines, as aquaponics is a game changer and is a better option than all other innovative methods, since it uses the waste from the fish advantageously compared to the natural eco system in which traditional agriculture happens. Aquaponics eliminates weeds and small animal excess, and it fully relies on recycling nutrient-rich water. Thus, there will be no toxicity compared to the conventional agricultural practices. Another major advantage is that aquaponics uses just a tenth of the amount of water required in conventional farming and doesn't use any harmful chemical and pesticides. Aquaponics can be set up and used anywhere, such as in green house and basement or in a drawing room; just by appropriate lighting and use of minimum space, any space can be utilized and converted into a garden that produces crops. This type of farming can be used for small-scale as well as medium-scale crop production. The system uses highly effluent fish food and produces nutrient-rich water which helps in optimum plant growth. Various types of aquaponics are used for the cultivation such as the deep water culture (DWC). DWC system is suitable for small-scale agriculture and for cultivating many fast-growing plants. With appropriate enhancements to the system design, it can also be used for both medium- and large-scale systems that yield larger agricultural produce that can be sold on a commercial scale to cater to more consumers. As discussed earlier, it can also be set up and used in-house to cultivate leafy green vegetables and herbs. Furthermore, the nutrient film technique needs a little more research and development to fine-tune and make it more robust in order to support the growth of plants and fruits such as strawberries and other herbs on an optimum level than is possible currently with the use of vertical aquaponics system. $\underline{14,15,16}$

\section{Conclusions}

The main challenge in the agricultural sector is to meet the demand of the growing food crisis along at the lowest cost possible. Given the cost considerations, the method to be chosen should be simple to use, efficient, and reliable. Our findings show that aquaponics can meet all these criteria. Just with any technology, this method have very limited issues and shortcomings, but the benefits clearly outweigh the disadvantages when compared to traditional agriculture. Other advantages of this system include the generation of nutrients that help boost plant and fish growth; as a result, in this system, expensive chemicals are replaced by less expensive fish feed, which means a major cost advantage in cultivation compared to traditional farming that requires more money and also the use of harmful chemicals and fertilizers that contaminate the crop and are harmful to food consumers. This system is thus closest to the natural ecosystem. The system produces zero waste, as it doesn't require discharging water, and is more productive compared to other agricultural techniques.

\section{Acknowledgement}

The manuscript is prepared by taking assistance from Accendere Knowledge Management Services Pvt. Ltd. We are thankful to them. We also express our gratitude to our teachers and mentor for guiding us throughout the work.

\section{References}

1. Roosta HR. Interaction between water alkalinity and nutrient solution $\mathrm{pH}$ on the vegetative growth, chlorophyll fluorescence and leaf magnesium, iron, manganese, and zinc concentrations in lettuce. J Plant Nutr. 2011;34(5):717-31.

2. Rakocy JE, Hargreaves JA. Nutrient accumulation in a recirculating aquaculture system integrated with hydroponic vegetable production. In: Techniques for modern aquaculture, proceedings of a conference, Sponkane; 1993. P. 148-58.

3. Saaid MF, Yahya NAM, Noor MZH, Ali MSAM. A development of an automatic microcontroller system for Deep Water Culture (DWC). In: 2013 IEEE 9th international colloquium on signal processing and its applications; 2013. P. 328-32.

4. McMurtry MR, Sanders DC, Cure JD, Hodson RG, Haning BC, Amand ECS. Efficiency of water use of an integrated fish/vegetable co-culture system. J World Aquacult Soc. 1997;28(4):420-28.

5. Graber A, Junge R. Aquaponic systems: Nutrient recycling from fish wastewater by vegetable production. Desalination. 2009;246(1-3):147-56. 
6. Sikawa DC, Yakupitiyage A. The hydroponic production of lettuce (Lactuca sativa L) by using hybrid catfish (Clarias microcephalus $\times$ C. gariepinus) pond water: potentials and constraints. Agric Water Manag. 2010;97(9):1317-25.

7. Rijn JV. Waste treatment in recirculating aquaculture systems. Aquacult Eng. 2013;53:49-56.

8. Tuhid NH, Abdullah NE, Khairi NM, Saaid MF, Shahrizam MSB, Hashim H. A statistical approach for orchid disease identification using RGB color. In: 2012 IEEE control and system graduate research colloquium.;2012. P. 382-5.

9. Martins CIM, Eding EH, Verdegem MCJ, Heinsbroek LTN, Schneider O, Blancheton J-P, et al. New developments in recirculating aquaculture systems in Europe: A perspective on environmental sustainability. Aquacult Eng. 2010;43(3):83-93.

10. Nhut DT, Huong MTN, Khiem D V, Da Silva JAT. Compact $3 \mathrm{U}$ as a novel lighting source for the propagation of some horticultural plants. J Appl Horticult. 2006;8(1):15-20.

11. Lessard JL, Hayes DB. Effects of elevated water temperature on fish and macro invertebrate communities below small dams. River Res Appl. 2003;19(7):721-32.
12. Saaid MF, Fadhil NSM, Ali MSAM, Noor MZH. Automated indoor aquaponic cultivation technique. In: 2013 IEEE 3rd international conference on system engineering and technology; 2013. P. 285-9.

13. Bear EA. Effects of temperature on survival and growth of west slope cutthroat trout and rainbow trout: implications for conservation and restoration. Montana State UniversityBozeman, College of Letters \& Science; 2005. P. 1-79.

14. Saaid MF, Zaini MFM, Noor MZH, Ali MSAM. Systematic data management of laboratory equipments using wireless technology. In: 2012 IEEE 8th International colloquium on signal processing and its applications; 2012. P. 316-20.

15. Saaid MF, Noor MZH, Ali MSAM, Rahim SA. A development of portable voice based device (PVBD) for stock identifications. In: 2013 IEEE 9th international colloquium on signal processing and its applications; 2013. P. 324-7.

16. Rajendra AB, Rajkumar N, Shreyas, V. 'Autonomous remote controlled car using machine learning, COMPUSOFT, An International Journal of Advanced Computer Technology, 2019; 8(8): 3307-3311. 